\title{
S'associer pour mieux rater. Duo auteur dramatique/metteur en scène et dynamique créative du rater réciproque
}

\section{Élise Deschambre}

\author{
(2) OpenEdition \\ Journals \\ Édition électronique \\ URL : https://journals.openedition.org/agon/8520 \\ DOI : 10.4000 /agon. 8520 \\ ISSN : 1961-8581 \\ Éditeur \\ Association Agôn \\ Référence électronique \\ Élise Deschambre, «S'associer pour mieux rater. Duo auteur dramatique/metteur en scène et \\ dynamique créative du rater réciproque », Agôn [En ligne], 9 | 2021, mis en ligne le 25 septembre 2021, \\ consulté le 17 janvier 2022. URL : http://journals.openedition.org/agon/8520 ; DOI : https://doi.org/ \\ 10.4000 /agon.8520
}

Ce document a été généré automatiquement le 17 janvier 2022.

Association Agôn et les auteurs des articles 


\title{
S'associer pour mieux rater. Duo auteur dramatique/metteur en scène et dynamique créative du rater réciproque
}

\author{
Élise Deschambre
}

1 Depuis l'avènement de la mise en scène en tant qu'art à part entière, l'auteur dramatique n'est plus la seule figure auctoriale reconnue du théâtre: le metteur en scène s'est imposé comme l'auteur de la représentation. En raison de ce dédoublement auctorial, écrivain de théâtre et metteur en scène ont souvent été renvoyés dos à dos. Comme le précise Camille Protar dans un article ${ }^{1}$ analysant différentes « rencontres » possibles du metteur en scène avec l'auteur, la mise en scène a rapidement utilisé le texte dramatique pour mieux s'affirmer en tant qu'art singulier : une représentation scénique s'impose comme œuvre en proposant une lecture spécifique et originale d'un texte dramatique. S'est alors instauré un rapport hiérarchique entre les figures de l'écrivain de théâtre et du metteur en scène qui, précisons-le, n'est pas sans lien avec la temporalité binaire ${ }^{2}$ de l'acte théâtral alors établie. Dans un cas, le metteur en scène impose sa lecture du texte et soumet ce dernier à un projet personnel dont il est le seul maître. Dans l'autre cas, le rapport s'inverse: l'auteur peut restreindre la liberté créatrice du metteur en scène et alors imposer son autorité créatrice. Protar donne l'exemple de l'«apparition foisonnante », dans les années 1980, « d'écritures nouvelles (Philippe Minyana, Michel Vinaver, Jean-Luc Lagarce, Didier Georges Gabily...) », « des écritures qui souffrent mal une prise trop grande de liberté tant le metteur en scène doit d'abord se confronter à leurs singularités, être à l'écoute de leurs rythmes, de leurs structures afin de les donner à entendre au spectateur ${ }^{3}$ ». Mais cette volonté d'agir sur la représentation se retrouvait déjà chez Samuel Beckett lorsqu'il consignait chaque geste scénique dans ses Actes sans parole ou dans Comédie et Va-et-vient, tout comme chez Jean Genet lorsqu'il adressait à Roger Blin ${ }^{4}$ une série de prescriptions pour la mise en scène des Paravents. 
2 On reconnaît là le « malentendu fondateur » entre les deux figures créatrices dont parle Georges Banu ${ }^{5}$ dans sa contribution à l'ouvrage Avènement de la mise en scène / Crise du drame. Continuités, discontinuités. Ce désaccord intrinsèque tel que présenté par Banu souffre toutefois d'une vision dualiste voire manichéenne. Le chercheur le repère en effet dans le rejet de l'auteur dramatique des libertés parfois prises par les metteurs en scène, ce rejet se manifestant principalement chez des auteurs "à forte personnalité " qui « cultivent l'affrontement et réclament la soumission ${ }^{6}$ ». Or, ce malentendu peut également opérer lorsqu'un metteur en scène met en doute voire conteste certains choix dramaturgiques de l'auteur du texte qu'il met en scène. Par ailleurs - et nous le montrerons dans cet article -, ce malentendu ne prend pas toujours la forme d'une domination revendiquée, et n'est pas nécessairement vécu négativement par l'une ou l'autre partie. La réflexion de Banu a néanmoins le mérite de mettre en avant ce rapport de tension artistique constante entre l'écrivain de théâtre et le metteur en scène : chacun d'eux dépend de l'œuvre de l'autre tout en ayant une œuvre propre à faire valoir. Autrement dit, les relations entre les deux figures reposent sur une tension entre « l'interdépendance et l'autonomie ${ }^{7}$ » de leurs arts.

3 Il n'est toutefois pas rare de voir s'associer un auteur dramatique et un metteur en scène. Pensons aux couples Anton Tchekhov/Constantin Stanislavski ou, plus récemment, Bernard-Marie Koltès/Patrice Chéreau. La réalité de telles réunions artistiques reste souvent dissimulée derrière les objets finaux ${ }^{8}$ que sont la représentation scénique et le texte entendu et éventuellement publié. Une perspective d'analyse génétique permet de mettre au jour de telles associations. Des travaux ${ }^{9}$ de génétique théâtrale ont notamment permis d'éclairer les relations des deux paires précédemment citées. À leur lecture, on constate que ces deux associations d'auteurs dramatiques et de metteurs en scène, si elles ont pu entrainer une certaine influence esthétique bilatérale, ne déplacent pas véritablement le rapport hiérarchique entre les deux figures créatrices: les œuvres textuelle et scénique ne sont pas pensées conjointement au sein d'un projet commun, et le metteur en scène et son projet prennent bien souvent le dessus, en témoignent les réserves de Tchekhov face à certains choix scéniques de Stanislavski et l'agacement qu'a fini par manifester Koltès face aux traitements scéniques de ses textes par Chéreau.

4 Le présent article entend identifier et interroger une autre forme d'association entre auteur dramatique et metteur en scène: une association où processus d'écriture dramatique et processus de création scénique, s'ils sont toujours envisagés dans leur autonomie artistique, se rencontrent et sont menés ensemble en vue d'un projet commun. Un tel partenariat suppose une dynamique relationnelle spécifique, basée sur des échanges fréquents sinon constants entre les deux figures créatrices. Il suppose également un mode de création qui s'appuie sur des essais, des modifications, des abandons mutuels. Le geste de création reposerait ainsi sur du rater, ce dernier étant moins à considérer comme une action (ratage) ou un état arrêté (raté) que comme un processus dynamique (concept du rater). Bien entendu, ce processus n'est pas absent des pratiques de mise en scène et d'écriture dramatique lorsqu'elles sont isolées l'une de l'autre. Lorsqu'il compose son texte, l'auteur écrit, rature, efface, réécrit. De même, le metteur en scène avance par tâtonnements, teste, revient sur des choix, en confirme d'autres. Le rater revêt alors une valeur créative et positive puisqu'il participe à l'élaboration de l'œuvre. Dans le cas qui nous occupe, le rater est toutefois singulier en ce qu'il est partagé et réciproque. Non seulement il est éprouvé aux niveaux tant de la 
matière textuelle que des intentions et choix scéniques, mais le rater de la matière artistique de chacune des deux figures est provoqué par l'évolution de la matière artistique de l'autre. Ce rater réciproque est donc le moteur de la création du spectacle. C'est l'interaction des deux champs artistiques qui fait progresser le travail à coup d'essais, de ratés et de relances.

Notre réflexion se construit à partir de deux cas d'étude : l'association entre l'auteur Jean-Marie Piemme et le metteur en scène Philippe Sireuil pour la création de Bruxelles, printemps noir (2018), et la collaboration de l'auteur Thomas Depryck et du metteur en scène Antoine Laubin au sein de leur compagnie commune, De Facto, pour la création de leur spectacle Dehors (2012). Dans les deux cas, le partenariat artistique s'appuie à la fois sur une relation amicale qui lie les deux $\operatorname{artistes}^{10}$ et sur une sensibilité théâtrale et esthétique partagée. De fait, Piemme et Sireuil sont tous deux adeptes d'un théâtre où la langue "boxe ${ }^{11}$ » et nécessite d'être travaillée (sur la page comme sur la scène) comme un matériau à part entière, où l'acteur se positionne dans le commentaire de la fiction plus que dans l'incarnation et où les questions d'actualité sont affrontées par le détour bienvenu de la fiction. En ce qui concerne Depryck et Laubin, l'un comme l'autre entendent faire du théâtre un lieu qui affronte, sans cynisme, défaitisme ou un tropplein d'empathie, les impasses de l'individu occidental contemporain et du système dans lequel il s'inscrit; d'un point de vue esthétique, tous deux portent une attention accrue à la littérarité de la langue et mettent en jeu des situations fictionnelles à la théâtralité fortement affichée. Parce qu'ils encouragent la confiance et l'écoute et facilitent le compromis au sein du couple artistique, ces liens amicaux, ces affinités esthétiques et ces partages d'une visée théâtrale favorisent assurément un mode de création par rater réciproque.

6 Si l'objet de cette étude appelle une perspective génétique, il ne sera toutefois pas question d'une étude génétique complète des textes et des représentations. Nous entendons bien plus révéler des pratiques de création avec l'aide d'outils de la génétique théâtrale. Notre analyse repose sur différents états des textes de chaque spectacle ainsi que, dans le cas de l'association Depryck/Laubin, sur de nombreux documents de travail du metteur en scène et, dans le cas de l'association Piemme/ Sireuil, sur nos notes et souvenirs personnels à propos de la création du spectacle pour lequel nous avons été assistante stagiaire à la mise en scène ${ }^{12}$. Des entretiens menés avec les deux auteurs ${ }^{13}$ ainsi que certains propos des deux metteurs en scène ${ }^{14}$ viennent l'étayer.

\section{Jean-Marie Piemme et Philippe Sireuil : la création de Bruxelles, printemps noir}

7 Le 22 mars 2016, deux bombes explosent à l'aéroport de Bruxelles-National et une autre dans une rame de métro de la capitale belge. Sireuil est alors occupé à travailler, avec ses élèves suisses des Teintureries, le texte inédit Métro 4. Écrit par Piemme en 2007, ce texte fait à l'époque écho aux attentats de Madrid et de Londres, et traite en une multitude de séquences indépendantes l'explosion fictive d'une bombe dans le métro bruxellois. Touché par les attentats de Bruxelles et ne pouvant nier l'étrange rencontre de la réalité et de la fiction, Sireuil propose à Piemme, qu'il connaît bien pour l'avoir eu comme dramaturge avant de monter une quinzaine de ses pièces, de retravailler le texte. De cette proposition naît tout d'abord une version intermédiaire jouée par les 
élèves de Lausanne en juin 2016, composée de séquences de Métro 4 (parfois retravaillées) et de quelques nouvelles scènes écrites après les attentats. Le projet sort ensuite de la sphère pédagogique et donne lieu à Bruxelles, printemps noir, spectacle joué au Théâtre des Martyrs (Bruxelles) en mars $2018^{15}$ dont le texte est publié quelques mois plus tard dans la collection Espace Nord ${ }^{16}$. Avec ce nouveau projet commun, Piemme et Sireuil souhaitent se saisir des attentats par les moyens du théâtre afin d'en faire voir les impacts sous de multiples perspectives, laissant au spectateur le soin de se situer dans l'événement et de trouver dans la fiction les outils d'une possible « réparation symbolique ${ }^{17}$ ».

8 Bien avant que les répétitions professionnelles ne débutent en janvier 2018, l'auteur et le metteur en scène échangent régulièrement autour de leur projet. Ces discussions visent la révision des enjeux du texte et de la mise en scène au regard de la nouvelle réalité tangible et traumatique des attentats, qui ne peut être niée. Les attentats font notamment rater une série de scènes et d'idées initiales. Plusieurs séquences de Métro 4 dont l'action se déroule dans les minutes qui suivent les explosions ont par exemple été reconsidérées et condensées dans la nouvelle séquence «Blessures». Leur enjeu similaire - dépeindre le choc et la variété des réactions de personnes qui étaient à proximité des bombes - autorise ce regroupement. De cette manière, Piemme et Sireuil évitent la redondance et, simultanément, font de la place pour de nouvelles séquences afin de multiplier les lieux, les temporalités et les points de vue ${ }^{18}$. En effet, de ces échanges provient, entre autres, la décision de présenter le point de vue d'un djihadiste. La séquence " J'aime la mort » voit ainsi le jour, Piemme y donnant la parole à un djihadiste fictif au travers d'une interview que visionnent une journaliste et ses collègues. S'ensuit la volonté partagée de dépeindre la communauté musulmane sous d'autres traits que ceux des terroristes. Piemme écrit alors «Les Enfants»: on y découvre Alia, dont le voile, la religion et les origines la confrontent, après les attentats, à la peur et au rejet d'une famille dont elle garde les enfants. L'événement réel des attentats fait donc rater plusieurs choix et intentions, mais mène l'auteur et le metteur en scène vers d'autres pistes.

Ces discussions entre Piemme et Sireuil se poursuivent durant la période des répétitions au début de l'année 2018, l'auteur assistant d'ailleurs à quelques-unes d'entre elles. Les répétitions sont pour Sireuil l'occasion de se frotter de façon concrète aux nouvelles séquences écrites par Piemme. Et certaines de ses intentions de mise en scène, au contact du texte, ratent et se précisent à la fois. Le cas de "J'aime la mort " est, à ce titre, significatif. Lors du travail de la séquence avec les acteurs, la prégnance des événements de Bruxelles qui transparaît dans le dialogue apparaît trop circonstancielle aux yeux de Sireuil. Celui-ci réalise qu'il souhaite mettre en lumière les mécanismes qui sous-tendent un acte terroriste plutôt que les motivations particulières des terroristes de Bruxelles, ce qui permet également de ne pas identifier le personnage $\mathrm{du}$ djihadiste à une personne réelle et de toujours se situer sur le versant fictionnel. Aussi propose-t-il de retirer les référents précis aux attentats de Bruxelles, ce que Piemme accepte volontiers, rejoignant l'avis du metteur en scène. Dans le même sens, les esquives du djihadiste et les marques d'affect de la journaliste sont retirées afin de ne pas placer l'échange des personnages sous l'angle du conflit interpersonnel et de mieux laisser transparaître le point de vue d'un djihadiste raisonné, coopérant et calme. 
10 Un autre exemple intéressant concerne la séquence «Les Hommes d'Etat » (intitulée par après «L'Honneur du Royaume » pour la version publiée). Issue des discussions préalables entre Piemme et Sireuil, cette séquence est écrite par l'auteur afin de mettre en jeu le point de vue de dirigeants politiques. Ces derniers sont représentés par Piemme comme des personnalités enfantines voire guignolesques («Les étrangers, caca. ", BPN p. 23). Sireuil décide alors de donner à voir les personnages non pas comme des hommes et femmes politiques réalistes, mais comme des guignols. Il affuble les acteurs (Jean-Pierre Baudson, Frank Arnaudon et Patrick Donnay) de têtes amplifiées, agrémentées de becs d'oiseaux carnassiers, à l'effigie de trois véritables ministres belges. Ce choix de Sireuil entraîne par la suite une réécriture majeure de la scène par Piemme. Tandis que la première version avait été écrite sans référent particulier, l'auteur, pour garantir une certaine cohérence de la situation, écrit une deuxième version qui s'appuie sur les convictions et désaccords politiques réels des différents ministres choisis. La première proposition dramaturgique de Piemme, qui est rédigée sous un prisme entièrement fictionnel, rate puisqu'elle ne convient plus à la situation mise en jeu par Sireuil; elle nécessite désormais l'insertion subtile d'éléments référentiels.

11 Les deux exemples cités, s'ils montrent comment les intentions de mise en scène de Sireuil peuvent rater et évoluer au contact des nouveaux textes de Piemme, montrent également comment le texte peut rater et se modifier au contact de nouveaux choix scéniques. Bien qu'il soit majoritairement constitué lorsque le travail au plateau débute, le texte ne cesse effectivement pas d'évoluer au rythme des répétitions et des nouvelles réflexions que ces dernières suscitent. Ces questions peuvent d'ailleurs prendre appui sur des discussions menées avec des comédiens, les échanges entre Piemme et Sireuil s'élargissant à plusieurs reprises au groupe d'acteurs. Ainsi, des suites d'une conversation entre l'acteur Ben Hamidou, l'auteur et le metteur en scène lors d'une répétition, l'Homme ensanglanté d'origine maghrébine de la séquence « La conférence de presse » affirmera tenir une librairie et non plus une épicerie (BPN p. 61). La discussion a en effet révélé l'emploi d'un stéréotype - association entre les Maghrébins habitant à Bruxelles et l'emploi d'épicier - et, par la même occasion, fait rater ce stéréotype, Piemme et Sireuil souhaitant s'extraire de leur point de vue et leur "réflexe d'"homme blanc"19 " pour aborder la question des attentats. Les modifications des enjeux du spectacle procèdent donc tantôt de propositions de Piemme, tantôt de suggestions de Sireuil, tantôt - bien que plus rarement - de réflexions de comédiens. Dans tous les cas, les avis et l'accord de l'auteur et du metteur en scène font loi. Cela souligne le contrôle que Piemme garde sur sa production même durant l'étape de création au plateau, bien que son geste d'écriture soit ouvert à la logique de la création scénique $^{20}$.

12 La description du processus de création de Bruxelles, printemps noir fait ainsi ressortir l'influence successive des choix artistiques de l'une des deux figures auctoriales sur le travail de l'autre. Certains choix du metteur en scène font effectivement rater et évoluer des choix dramaturgiques de l'auteur, et certains choix dramaturgiques de l'auteur font rater et évoluer des choix scéniques du metteur en scène. Qui plus est, elle nous permet de préciser le type de collaboration qui unit Piemme et Sireuil. Leur travail collaboratif tient de l'adaptation : l'auteur aménage son texte selon l'évolution du projet scénique et de sa logique, et le metteur en scène adapte son projet de création scénique selon l'évolution du texte et de sa logique. 


\section{Thomas Depryck et Antoine Laubin (De Facto) : la création de Dehors}

13 Laubin et Depryck travaillent ensemble au sein de la compagnie De Facto ${ }^{21}$ dont ils forment le noyau, Laubin animant seul la compagnie avec la complicité de l'auteurdramaturge depuis le succès des Langues paternelles (2009). À l'origine du spectacle Dehors se trouve la volonté des deux artistes d'aborder la question des personnes sans domicile fixe et de la «désocialisation» dans ce qui leur semble être une «juste distance $^{22}$ " théâtrale, c'est-à-dire sans opter pour un théâtre documentaire ou journalistique, ni verser dans un théâtre moralisateur ou faussement empathique. Ils entendent interroger leur malaise, leur colère et leur impuissance face à la question et mettre en lumière ce que révèlent à leurs yeux les « désocialisés » de l'individu et de la société d'aujourd'hui.

14 Le metteur en scène et l'auteur s'engagent alors dans un processus de recherche et de travail de plusieurs années, dont le temps fort sera leur résidence, en compagnie de six acteurs, de quinze semaines (réparties sur trois années, de septembre 2008 à mai 2011) au sein du Théatre de L'L, espace de recherche et d'accompagnement dédié à la jeune création en arts vivants situé à Bruxelles. Si ces étapes de travail n'avaient pas pour obligation la création d'un spectacle, le temps d'expérimentation accordé par L'L n'étant pas conditionné par l'obtention de quelconques résultats, elles ont néanmoins abouti à un spectacle créé au Théâtre Royal de Namur en octobre $2012^{23}$ dont le texte a été publié par les éditions Lansman en partenariat avec L'L et le Centre des Écritures Dramatiques Wallonie-Bruxelles (CED-WB) ${ }^{24}$. Dans cette forme spectaculaire finale, la question de la désocialisation est traitée au moyen d'un effet de métathéâtralité : six acteurs, qui se montrent comme tels sur scène et sont désignés comme tels dans le texte, s'attaquent au sujet de la marginalisation sociale (se mettre ou être mis au dehors du cadre social), alternant scènes de fiction et temps de réflexions personnelles.

Durant le long parcours de recherche et de travail, qu'on peut rétrospectivement désigner comme le processus de création du spectacle, l'équipe partage des lectures (les livres Les Naufragés et Le Sang nouveau est arrivé de Patrick Declerck qui ont initié le projet, mais aussi des coupures de presse, des témoignages...), s'adonne à des réflexions collectives et rassemble divers textes de théâtre qui, dans un premier temps du moins, semblent prolonger l'interrogation initiale de Laubin et Depryck. De là vont se développer la détermination de la forme de la représentation et la composition du texte. Réflexions, expérimentations scéniques et écriture sont intimement liées. Laubin le souligne dans la préface du texte édité : " C'est un spectacle que nous avons "conçu" à huit, et les mots de Thomas Depryck en constituent le socle littéraire, écrits en dialogue étroit avec le travail collectif. ${ }^{25}$ "

16 Tout au long du processus, Depryck écrit en effet plusieurs situations fictionnelles à partir des textes rassemblés et des sujets et questions soulevés par l'équipe. Les séquences qui émanent de sa production sont alors essayées au plateau: tous s'y confrontent, y réagissent, des impasses apparaissent, d'autres voies empruntées se confirment... Par exemple, dès la première semaine de travail à L'L, durant laquelle le groupe éprouve au plateau un extrait d'un des textes théâtraux préalablement choisis (Ma Solange, comment t'écrire mon désastre, Alex Roux de Noëlle Renaude) et un texte écrit par Depryck à partir de cet extrait, les comédiens et Laubin constatent que le texte de 
Renaude ne «fonctionne » pas comme espéré. Leur première idée rate. En revanche, le texte de Depryck "réussit» en ce qu'il s'approche davantage de ce que le groupe entend exprimer. L'équipe prend alors la décision de ne plus travailler les textes des autres auteurs et de se focaliser sur la production de Depryck. Des textes exogènes ne demeureront que des thèmes ou des situations réécrites par Depryck.

À plusieurs reprises, des improvisations sont également réalisées par les acteurs à partir de suggestions de Laubin. Depryck observe alors l'exercice en cours puis, dans un deuxième temps, compose différents textes à partir des propositions scéniques. Ces textes retournent par la suite au plateau pour être mis à l'essai et, à partir du travail qu'en font les acteurs et Laubin, Depryck les retravaille, en écrit d'autres, et ainsi de suite. Ainsi, les textes "Patrick 1 », «Patrick 2 » et "Patrick 3 » (Dehors p. 18-19, p. 46-47 et p. 64), où "Ca" (pour l'actrice Caroline Berliner) s'adresse à Patrick Declerck, ont été nourris par des improvisations. Il en va de même pour la séquence «Les clochards » dans laquelle une haine des SDF est passée au crible par celui qui la porte («Je hais les clochards parce qu'ils [...]», «Je hais les clochards parce que [...]», Dehors p. 63). Le principe de l'improvisation va être également conservé comme tel dans le spectacle, Depryck ne fixant pas l'entièreté du texte. De fait, non seulement l'ordre des scènes dépend d'un tirage au sort effectué sur le plateau (la version éditée est donc une solution d'ordonnancement parmi d'autres, et n'y figurent pas les "scènes jaunes ${ }^{26} "$, quatre conclusions musicales possibles dont une est tirée au sort à chaque représentation), mais plusieurs séquences sont destinées à être improvisées par les acteurs au cours de la représentation. Il en va ainsi des « scènes rouges ", une vingtaine de questions autour de la clochardisation que les comédiens choisissent au hasard et s'adressent, chacun devant répondre en son nom. Dans ce dernier cas, les questions ont été mises sur papier par Depryck à partir d'improvisations réalisées pendant le processus de création. Treize d'entre elles figurent dans la version éditée.

18 Le processus de création a donc généré une grande quantité de propositions scéniques et textuelles dont beaucoup ont été abandonnées. La tentative infructueuse de rejouer une interview de Declerck pour l'émission télévisée « Noms de dieux » est un exemple significatif. L'essai a offert un premier résultat qui ne convenait pas au groupe; il a, en ce sens, raté, et la séquence a été abandonnée. Il a toutefois laissé place à l'idée d'une émission fictive de débat télévisé entre des invités dont aurait fait partie Declerck, idée qui a généré plusieurs improvisations et versions textuelles. Cette entreprise a elle aussi raté. Mais elle a engendré de nouveaux principes scéniques et de nouveaux textes : l'idée du débat a été gardée mais déplacée dans le réel de la représentation par le biais des "scènes rouges "; les personnages du débat télévisé sont devenus les rôles centraux de plusieurs scènes écrites par Depryck ("La ministre ", "Le journaliste ", "Le travailleur social », «Le type »); et la présence de Declerck, maintenue un temps de manière directe (une actrice parle au nom de Declerck), est devenue indirecte (une actrice s'adresse à Declerck). Remarquons que cette succession de ratés permet à l'équipe de progresser vers une forme qui soit plus en phase avec son objectif initial, qui est d'interroger la marginalisation sociale depuis les points de vue et questionnements singuliers et parfois divergents de ses membres. Effectivement, non seulement les artistes s'éloignent d'un théâtre à vocation documentaire (présent dans la tentative de reconstituer l'interview), mais le propos est désormais traité davantage par la médiation des acteurs qui, s'ils jouent bel et bien plusieurs rôles fictionnels, ne se cachent pas derrière la fiction, parlant même parfois en leurs noms propres. 
Si elles sont complémentaires, l'écriture du texte et la mise au point scénique restent envisagées ici comme deux gestes artistiques autonomes. En effet, même si Depryck puise matière dans le travail scénique (que ce soit en réécrivant son texte en fonction du travail qui est fait sur le plateau ou en écrivant à partir d'improvisations), son acte d'écriture comme son texte s'inscrivent dans une certaine indépendance artistique. Lors des conversations, lors des improvisations ou lors des essais au plateau, il ne prend pas scrupuleusement note et n'enregistre rien. Il garde bien plutôt une idée, une impression de ce qui a été dit et a été fait à partir de laquelle il déploie ensuite son propre savoir-faire d'écrivain, retravaillant plus que retranscrivant ce qu'il a vu ou entendu au plateau.

La publication du texte du spectacle confirme cette autonomie. Certes, le livre est marqué par la représentation originellement liée au texte, au travers notamment de la préface contextualisante de Laubin, mais aussi du choix de référer aux acteurs, énonciateurs scéniques des propos, et non pas aux personnages, énonciateurs fictionnels des propos. Ainsi, Berliner ( $\mathrm{Ca} »$ dans le corps du texte) assume divers rôles, comme l'indique une note liminaire :

Caroline Berliner (Ca) : Louise, L'assistante, Celle qui s'adresse à Patrick, Elle-même.

(Dehors p. 6)

21 Elle sera chaque fois indiquée comme locutrice, qu'elle endosse par exemple le rôle de Louise, femme désocialisée ( Ca : Il fait tout noir. Cette cellule n’a pas de murs. [...] Je m'y suis égarée et j'attends. », monologue inaugural de Louise, Dehors p. 15) ou celui de l'assistante d'autopsie ( $\mathrm{Ca}$ : Je ne sais pas comment on fait pour rester insensible à ça. Ce corps qui ne bouge plus.", scène de l'autopsie de John, un SDF, Dehors p. 37). Néanmoins, la publication du texte en fait un objet artistique à part entière qui existe aux côtés de la représentation. Ce faisant, le texte se "détache » du spectacle initial et s'ouvre à d'autres représentations scéniques possibles. C'est pourquoi l'acte de publication a été désiré par Depryck. Comme il nous l'a déclaré lors de notre entrevue, l'auteur a pensé l'édition de son texte de manière à ce qu'il puisse faire œuvre et qu'une autre équipe puisse s'en saisir (ce qui a partiellement été le cas avec le spectacle Pièces montées des élèves du Conservatoire du $8^{\mathrm{e}}$ arrondissement de Paris en 2014, où des extraits du texte ont été repris).

Par conséquent, s'ils ont été conçus ensemble, ni l'un ni l'autre n'ayant été déterminé $a$ priori, texte et représentation scénique restent deux objets artistiques distincts relevant de deux arts spécifiques, celui de l'écriture dramatique et celui de la mise en scène. Tous deux ont été élaborés l'un par rapport à l'autre, chacun ayant contribué à la genèse de l'autre. Le travail au plateau a fait avancer l'écriture autant que l'écriture a fait avancer le travail au plateau. Tantôt, c'est l'un qui est premier (lorsque les acteurs improvisent et que Depryck écrit ensuite à partir des improvisations), tantôt, c'est l'autre qui est première (lorsque Depryck propose des textes et que les acteurs et le metteur en scène les essaient sur le plateau). Dans tous les cas, le travail au plateau et l'écriture dramatique se succèdent tour à tour, se rencontrent, se répondent, s'interrogent, et se valident mutuellement ou se font rater et se relancent. Auteur et metteur en scène ne s'aventurent pas hors de leurs domaines artistiques respectifs mais suivent la gestation progressive de l'objet artistique dont ils n'ont pas la maîtrise. Chacun ajuste alors sa matière jusqu'à trouver une forme commune qui soit juste à leurs yeux. 

Depryck et Laubin, lui, tient plutôt d'un rapport d'appropriation : l'auteur trouve sur le plateau matière pour composer son drame et le metteur en scène trouve dans les textes qu'il met à l'essai matière pour construire son projet scénique. Cette collaboration sous forme d'appropriation est favorisée par le point de départ du projet. De fait, la problématique de la clochardisation et de la précarisation sociale a suscité une démarche dramaturgique spécifique de recherche collective d'un contenu et d'une forme adéquats qui n'existaient pas encore, la logique du texte et celle du plateau étant dès lors à inventer conjointement. Ce processus d'expérimentation et d'élaboration conjointe encourage particulièrement un procédé créatif fait de ratages mutuels et successifs.

\section{Dynamique créative du rater réciproque}

L'analyse des processus de création de Bruxelles, printemps noir et Dehors révèle des partenariats singuliers où le geste artistique d'une des deux figures auctoriales rend problématique celui de l'autre, et inversement. Régulièrement, écriture et mise en scène se font, d'une certaine façon, rater, et se relancent, se mènent vers d'autres tentatives. Écrivain et metteur en scène procèdent donc par tâtonnements mutuels et s'inscrivent dans une dynamique créative de rater réciproque : le spectacle final est rendu possible par plusieurs ratages textuels et scéniques qui se suivent et se répondent. L'écriture dramatique et la mise en scène ne fusionnent donc pas, pas plus qu'elles ne sont pensées en un seul mouvement. Chaque pratique se noue à l'autre sans s'y confondre, deux mouvements artistiques se rencontrent sans jamais coïncider tout à fait. Autrement dit, les deux figures font dialoguer leurs arts en vue d'un compromis plus qu'elles ne les fondent l'un dans l'autre. Chacun reste ainsi le maitre de son art : le rater est certes réciproque, mais chaque figure auctoriale assume celui de sa propre matière artistique.

De la sorte, les partenariats de Piemme et Sireuil et de Depryck et Laubin fonctionnent selon une dialectique artistique. La dualité entre les deux figures auctoriales devient la source de la création théâtrale: c'est de l'irréductibilité des pratiques d'écriture dramatique et de composition scénique que naît le spectacle. Le «malentendu fondateur " est donc pleinement assumé et devient une force, la tension artistique entre les deux figures est tournée en principe moteur et créateur. Mais ce mode de création n'impose pas pour autant une hiérarchie verticale. L'élaboration réciproque et dialectique du texte et de la forme scénique induit une relation artistique qui relève davantage d'une collaboration à tendance horizontale. En effet, les deux artistes s'ouvrent volontiers à la logique artistique de l'autre, et chaque figure fait rater la matière de l'autre. Tous deux doivent également pouvoir se déclarer satisfaits du résultat auquel leur rater les amène, sans quoi il serait question d'un raté supplémentaire susceptible de mener le processus de création vers d'autres possibilités.

Si elle ne fait donc pas fusionner les deux temps de la création théâtrale tels que définis par Henri Gouhier, cette pratique dialectique ne les maintient pas pour autant. Elle les multiplie, si nous reprenons l'idée de Joseph Danan ${ }^{27}$. Nous n'avons effectivement pas affaire à un temps de création suivi d'un temps d'exécution, mais à une multitude de temps de création et d'exécution qui se génèrent par ratages et se succèdent d'une telle façon qu'il est parfois laborieux d'en fixer les frontières. L'écriture peut par ailleurs 
enclencher le travail scénique autant que l'élaboration scénique peut enclencher l'écriture ; cette dernière n'est donc plus automatiquement un temps de création et le travail au plateau un temps d'exécution. Non seulement les temps établis par Gouhier sont multipliés, mais ils ne sont plus assignés à une pratique artistique en particulier. Cette multiplication des temps engendrée par le rater réciproque trouvera son terme lorsqu'un résultat sera ressenti comme juste par les deux figures auctoriales, sans qu'il soit toujours possible pour les artistes de le justifier. C'est effectivement le sentiment que quelque chose ne va pas, n'est pas juste (ce quelque chose étant plus ou moins identifié par les praticiens selon les cas) qui fait qu'il y a des ratés et qui relance la création $^{28}$.

Il semble que cette dynamique du rater réciproque soit de plus en plus éprouvée dans la pratique théâtrale contemporaine. Citons les paires artistiques que forment Ronan Chéneau et David Bobée, Kossi Efoui et Nicolas Saelens, Olivier Saccomano et Nathalie Garraud, ou Antonio Rojano et Víctor Velasco à certaines occasions. Dans chaque cas, auteur dramatique et metteur en scène s'associent au nom d'un projet commun et procèdent par essais et ratages mutuels, la matière artistique de chacun évoluant au contact de celle de l'autre. Un duo plus ancien mérite toutefois d'être cité, celui formé par Jean Giraudoux et Louis Jouvet. Leur collaboration constitue certainement les prémisses des partenariats éclairés dans cet article. En effet, Giraudoux assistait aux répétitions dirigées par Jouvet et n'hésitait pas à modifier son texte selon les nécessités du projet scénique. Il faudrait cependant s'interroger sur la réciprocité de cette collaboration : si Giraudoux s'ouvrait aisément à la logique du plateau et retravaillait son texte en fonction, Jouvet et lui s'inscrivaient-ils pour autant dans une dynamique de rater réciproque ? À en croire les termes de Protar, rien n'est moins sûr. Le travail de Giraudoux, affirme la chercheuse, " est dévoué à celui du metteur en scène ${ }^{29}$ ", là où dans les binômes évoqués plus haut auteur et metteur en scène sont, d'une certaine manière, dévoués l'un à l'autre.

Précisons néanmoins que bien qu'ils témoignent ensemble d'un processus dynamique de rater réciproque susceptible d'être observé chez d'autres duos, les partenariats qui lient Piemme et Sireuil d'un côté et Depryck et Laubin de l'autre ne sont pas identiques. Chacun des deux couples s'inscrit en effet dans une organisation d'ensemble distincte : Sireuil est au centre de la compagnie qui réalise le projet et invite Piemme à le rejoindre dans le cadre dudit projet, tandis que Laubin et Depryck font tous deux partie de la compagnie porteuse du projet artistique. Nous l'avons évoqué, la façon dont les auteurs et les metteurs en scène travaillent en fonction de l'objet artistique qu'ils ne maitrisent pas diffère également. Dans le cas Piemme/Sireuil, la collaboration s'organise sur un mode adaptatif; dans le cas Depryck/Laubin, elle s'organise sur un mode appropriatif. Ces distinctions pourraient s'avérer opérantes pour l'analyse d'autres partenariats basés sur un processus de rater réciproque, entre autres ceux cités plus haut. Le lien éventuel entre la configuration d'ensemble dans laquelle le duo auteur/metteur en scène s'inscrit et la nature de la relation qui unit le geste d'écriture et le geste de création scénique reste à interroger, notamment à partir d'un plus large panel de couples artistiques.

Cette dynamique créative du rater réciproque demande également qu'on se penche davantage sur le statut symbolique des deux figures auctoriales. En effet, l'auteur dramatique voit son statut modifié par la position artistique particulière qu'il occupe, s'inscrivant au cœur du projet et du processus de création scénique tout en s'y 
démarquant en tant que spécialiste de la production textuelle, et gardant la pleine maitrise sur son texte tout en le faisant rater et évoluer au contact de la logique du plateau. Et qu'en est-il de la position et fonction du metteur en scène lorsqu'il s'inscrit dans cette dynamique? Si dans son article Protar évoque la possibilité d'une telle collaboration à travers l'exemple de Saccomano et Garraud, elle ne développe cependant pas la question de l'impact que peut avoir cette collaboration sur le statut symbolique du metteur en scène. Il est par ailleurs évident que l'acteur joue un rôle prépondérant dans les pratiques que nous avons décrites ici. Même s'il n'a pas le dernier mot, il occupe une place essentielle dans la création du spectacle et va au-delà de l'interprétation «technique» du jeu dramatique: il suscite des ratés et des modifications et, en cela, participe au rater de l'auteur dramatique et/ou du metteur en scène. Le couple auctorial s'inscrit donc dans une dynamique de groupe plus large qu'il conviendrait de ne pas occulter, les autres artistes de la création du spectacle étant susceptibles de jouer un rôle dans le rater réciproque qui unit les deux figures, ce qui ne va pas sans complexifier la question de l'auctorialité et des rapports hiérarchiques.

\section{BIBLIOGRAPHIE}

AUTANT-MATHIEU, Marie-Christine, « Tchekhov / Stanislavski ou la naissance de la mise en scène. Du texte dramatique à la partition scénique », in Almuth Grésillon et al. (dir.), Genèses théâtrales, Paris, CNRS, 2010, p. 179-194.

BANU, Georges, «L'auteur et le metteur en scène : un malentendu qui dure ", in Jean-Pierre Sarrazac, Marco Consolini (dir.), Avènement de la mise en scène / Crise du drame. Continuitésdiscontinuités, Bari, Edizioni di Pagina, 2009, p. 237-244.

BENHAMOU, Anne-Françoise, "Genèse d'un combat : une rencontre "derrière les mots" ", in Genesis, $n^{\circ} 26$, "Théâtre », sous la dir. de Almuth Grésillon et Nathalie Léger, 2006, p. 51-69.

BOILLOT, Jean, et al., « Jean-Marie Piemme et ses metteurs en scène. Entretien avec Jean Boillot, Antoine Laubin, Philippe Sireuil et Virginie Thirion », propos recueillis par Pierre Piret, in Textyles, $n^{\circ}$ 60, «Jean-Marie Piemme. Quel théâtre pour le temps présent ? ", sous la dir. de Pierre Piret, 2021, p. 137-152.

DANAN, Joseph, « Un art à mille temps », in Rafaëlle Jolivet Pignon, La Représentation rhapsodique. Quand la scène invente le texte. Simon McBurney, Romeo Castellucci, Pippo Delbono, Christoph Marthaler, François Tanguy, Montpellier, L'Entretemps, 2015, p. 9-16.

DEPRYCK, Thomas, Dehors suivi de Le réserviste, Carnières-Morlanwelz, Lansman éditeur / CEDWB / L'L, 2013.

DESCHAMBRE, Élise, « Dire l'(après) attentat : enjeux formels de Bruxelles, printemps noir », in Textyles, $n^{\circ}$ 60, «Jean-Marie Piemme. Quel théâtre pour le temps présent ? , sous la dir. de Pierre Piret, 2021, p. 73-86.

GOUHIER, Henri, Le théâtre et les arts à deux temps, Paris, Flammarion, 1989. 
HAMON-SIRÉJOLS, Christine, « Tchékhov / Stanislavski : un dialogue artistique complexe », in Jean-Pierre Sarrazac, Marco Consolini (dir.), Avènement de la mise en scène / Crise du drame. Continuités-discontinuités, Bari, Edizioni di Pagina, 2009, p. 263-274.

LAUBIN, Antoine, « Regards sur Dehors », in Thomas Depryck, Dehors suivi de Le réserviste, Carnières-Morlanwelz, Lansman éditeur / CED-WB / L'L, 2013, p. 7-8.

LAUBIN, Antoine, « Résidence : Dehors », in Scènes, n 24, 2009, p. 53-55.

PIEMME, Jean-Marie, Bruxelles, printemps noir suivi de Scandaleuses et 1953, Bruxelles, Impressions nouvelles, coll. « Espace Nord », 2018.

PROTAR, Camille, « Écritures plurielles, écritures scéniques. Rencontres du metteur en scène avec l'auteur ", in Izabella Pluta (dir.), Metteur en scène aujourd'hui. Identité artistique en question?, Rennes, Presses Universitaires de Rennes, 2017, p. 33-47.

SIREUIL, Philippe, « Piemme écrit comme on boxe », propos recueillis par Bernard Debroux, in Alternatives théâtrales, $\mathrm{n}^{\circ}$ 75, « Jean-Marie Piemme », 2002, p. 35-37.

\section{NOTES}

1. Camille Protar, "Écritures plurielles, écritures scéniques. Rencontres du metteur en scène avec l'auteur ", in Izabella Pluta (dir.), Metteur en scène aujourd'hui. Identité artistique en question?, Rennes, Presses Universitaires de Rennes, 2017, p. 33-47.

2. Pour approfondir, voir Henri Gouhier, Le théâtre et les arts à deux temps, Paris, Flammarion, 1989.

3. Camille Protar, op. cit., p. 36.

4. Jean Genet, Lettres à Roger Blin, Paris, Gallimard, 1966.

5. Georges Banu, "L'auteur et le metteur en scène : un malentendu qui dure ", in Jean-Pierre Sarrazac, Marco Consolini (dir.), Avènement de la mise en scène / Crise du drame. Continuitésdiscontinuités, Bari, Edizioni di Pagina, 2009, p. 237-244.

6. Ibid., p. 240.

7. Camille Protar, op. cit., p. 36.

8. Bien qu'au théâtre l'existence d'un «objet final " soit problématique, le spectacle (texte y compris) pouvant varier d'une représentation à l'autre, et le texte publié étant toujours susceptible d'être réactualisé et modifié dans un nouveau projet de mise en scène.

9. Marie-Christine Autant-Mathieu, «Tchekhov / Stanislavski ou la naissance de la mise en scène. Du texte dramatique à la partition scénique », in Almuth Grésillon et al. (dir.), Genèses théâtrales, Paris, CNRS, 2010, p. 179-194; Anne-Françoise Benhamou, « Genèse d'un combat : une rencontre "derrière les mots" ", in Genesis, n 26, "Théâtre ", sous la dir. de Almuth Grésillon et Nathalie Léger, 2006, p. 51-69; Christine Hamon-Siréjols, « Tchékhov / Stanislavski : un dialogue artistique complexe », in Jean-Pierre Sarrazac, Marco Consolini (dir.), op. cit., p. 263-274.

10. L'amitié entre Piemme et Sireuil naît de leur travail de collaboration, Piemme ayant été le dramaturge de Sireuil durant plusieurs années avant d'écrire et d'être monté par le metteur en scène. Laubin et Depryck ont, quant à eux, noué un lien amical lors de leurs études communes.

11. Sireuil, Philippe, « Piemme écrit comme on boxe ", propos recueillis par Bernard Debroux, in Alternatives théâtrales, $\mathrm{n}^{\circ}$ 75, «Jean-Marie Piemme », 2002, p. 35-37.

12. Nous tenons à remercier chaleureusement Thomas Depryck, Jean-Marie Piemme, Antoine Laubin et Philippe Sireuil de nous avoir transmis ces différents documents.

13. Entretiens menés dans le cadre de notre recherche doctorale les 24 (Piemme) et 30 (Depryck) juin 2020 à Bruxelles. Inédits. 
14. Pour Sireuil, nous renvoyons à son intervention à la table ronde des metteurs en scène organisée dans le cadre de la journée d'étude «Jean-Marie Piemme : quel théâtre pour le temps présent?" à Louvain-la-Neuve le 29 novembre 2018, reprise dans le numéro 60 de la revue Textyles (2021). Pour Laubin, nous nous référons à la préface du texte du spectacle édité (Lansman, 2013) et à un article rédigé par le metteur en scène dans la revue Scènes $\left(\mathrm{n}^{\circ} 24,2009\right.$, p. 53-55).

15. Bruxelles, printemps noir, mise en scène de Philippe Sireuil, texte de Jean-Marie Piemme, 2018, coproduction La Servante / Théâtre en Liberté / Théâtre National Wallonie-Bruxelles / Compagnie Biloxi 48, avec l'aide du Centre des Arts Scéniques. Assistanat à la mise en scène : Delphine Peraya, aidée par Manon Andréo et Élise Deschambre. Scénographie : Vincent Lemaire. Lumières : Benoit Théron. Création sonore : Geoffrey Sorgius. Vidéo : Stefano Serra. Costumes : Catherine Somers, assistée de Pauline Miguet. Avec Frank Arnaudon, France Bastoen, Jean-Pierre Baudson, Isabelle De Beir, Dolorès Delahaut, Sophie Delogne, Patrick Donnay, Soufian El Boubsi, Itsik Elbaz, Maude Fillon, Janine Godinas, Ben Hamidou, Agathe Hauser, Antoine Herbulot, Daniel Jeanloz, Charlotte Leblé, Stéphane Ledune, Fabrice Rodriguez et Laurent Tisseyre.

16. Jean-Marie Piemme, Bruxelles, printemps noir suivi de Scandaleuses et 1953, Bruxelles, Impressions nouvelles, coll. «Espace Nord ", 2018. Tout renvoi à cette édition sera dorénavant abrégé BPN et indiqué entre parenthèses dans le texte.

17. Nous reprenons l'expression du sous-titre de Rwanda 94, texte co-écrit par Piemme pour le Groupov.

18. Pour une analyse dramaturgique approfondie de la réécriture et de ses enjeux, voir Élise Deschambre, «Dire l'(après) attentat: enjeux formels de Bruxelles, printemps noir ", in Textyles, $\mathrm{n}^{\circ}$ 60, «Jean-Marie Piemme. Quel théâtre pour le temps présent?», sous la dir. de Pierre Piret, 2021, p. 73-86.

19. Propos de Sireuil à l'occasion de la journée d'étude «Jean-Marie Piemme : quel théâtre pour le temps présent ? ", repris dans Textyles, $\mathrm{n}^{\circ}$ 60, «Jean-Marie Piemme. Quel théâtre pour le temps présent?», sous la dir. de Pierre Piret, 2021, p. 146.

20. Remarquons que cette ouverture à la logique du plateau est affirmée par Piemme au cœur même de son texte, bien avant les répétitions : l'auteur laisse l'organisation des différentes séquences, à l'exception des deux premières et de la dernière, à la discrétion du metteur en scène (l'ordre qui figure dans la version publiée est celui déterminé par Sireuil pour sa mise en scène).

21. Depryck écrit également en dehors du cadre de De Facto, collaborant avec d'autres metteurs en scène ou collectifs ou écrivant seul des textes qui ne sont pas liés à des projets scéniques précis.

22. Antoine Laubin, « Résidence : Dehors ", in Scènes, $n^{\circ} 24,2009$, p. 53.

23. Dehors, mise en scène d'Antoine Laubin, texte de Thomas Depryck, 2012, production De Facto asbl, coproduction Théâtre de Namur / Maison de la Culture de Tournai, avec l'aide du Ministère de la Communauté française - Service du Théâtre. Assistanat à la mise en scène : Christelle Alexandre, aidée par Léa Tarral. Scénographie et costumes : Aurélie Forges. Lumières : Gaspard Samyn. Vidéo: Sung-A Yoon. Avec Caroline Berliner, Coraline Clément, Denis Laujol, Jérôme Nayer, Hervé Piron et Renaud Van Camp.

24. Thomas Depryck, Dehors suivi de Le réserviste, Carnières-Morlanwelz, Lansman éditeur / CEDWB / L'L, 2013. Tout renvoi à cette édition sera dorénavant abrégé Dehors et indiqué entre parenthèses dans le texte.

25. Antoine Laubin, « Regards sur Dehors », in Thomas Depryck, op. cit., p. 7.

26. Ibid., p. 8.

27. Joseph Danan, "Un art à mille temps ", in Rafaëlle Jolivet Pignon, La Représentation rhapsodique. Quand la scène invente le texte. Simon McBurney, Romeo Castellucci, Pippo Delbono, Christoph Marthaler, François Tanguy, Montpellier, L’Entretemps, 2015, p. 9-16. 
28. La contrainte temporelle de production peut, elle aussi, mettre un terme au rater réciproque. Le résultat final pourrait alors ne pas avoir rencontré cette exigence de justesse partagée et le processus de création s'arrêter sur un raté (perçu comme tel ou non par les spectateurs, mais nous touchons là à une autre question).

29. Camille Protar, op. cit., p. 34.

\section{RÉSUMÉS}

La présente contribution entend spécifier une forme singulière de partenariat entre les figures de l'auteur dramatique et $\mathrm{du}$ metteur en scène. Par une analyse descriptive du travail de collaboration de deux duos (Jean-Marie Piemme/Philippe Sireuil et Thomas Depryck/Antoine Laubin) pour la création de deux spectacles en particulier, elle dévoile des partenariats basés sur un processus créatif de rater réciproque et envisage les multiples prolongements critiques suscités par une telle dynamique collaborative.

\section{INDEX}

Mots-clés : auteur dramatique, metteur en scène, duo, partenariat, rater réciproque, processus de création, Piemme (Jean-Marie), Sireuil (Philippe), Depryck (Thomas), Laubin (Antoine)

\section{AUTEUR}

\section{ÉLISE DESCHAMBRE}

Élise Deschambre est doctorante au Centre d'études théâtrales de Louvain-la-Neuve en Belgique (bourse FRESH, F.R.S.-FNRS). Sa recherche doctorale entend identifier et théoriser un nouveau statut du texte et de l'auteur dramatiques au tournant des XXe et XXIe siècles. 